\title{
Sinais e sintomas de disfagia orofaríngea em idosos institucionalizados: revisão integrativa
}

\section{Signs and symptoms of oropharyngeal dysphagia in institutionalized older adults: an integrative review}

\author{
Stefane Maria de Lima Campos ${ }^{1}$ (D), Davi Reuel Pontes Trindade ${ }^{1}$ (D), Renata Veiga Andersen Cavalcanti ${ }^{1}$, \\ Karinna Verissimo Meira Taveira² (1D, Lidiane Maria de Brito Macedo Ferreira ${ }^{3}$ (D), \\ Hipólito Virgilio Magalhães Junior ${ }^{1}$ (1)
}

\section{RESUMO}

Objetivo: identificar quais são os sinais e sintomas de disfagia orofaríngea mais presentes nos idosos residentes em Instituições de Longa Permanência. Estratégia de pesquisa: revisão integrativa realizada em quatro bases de dados: Embase, LILACS, MEDLINE/PubMed e Web of Science, com uso de termos na língua inglesa e aplicação de filtros por idioma e idade. Critérios de seleção: estudos disponíveis na forma de texto completo em inglês, português ou espanhol, sem restrição de tempo de publicação, relacionados a idosos residentes em Instituições de Longa Permanência que referiram disfagia orofaríngea. Foram excluídos estudos relacionados a idosos da comunidade ou que estavam em hospitais, e com outras condições de saúde não relacionadas aos problemas de deglutição. Resultados: de 389 estudos, 16 foram incluídos nesta revisão, publicados entre os anos de 1986 e 2020. Houve predomínio de participantes do sexo feminino, com média mínima de idade de 71 anos e máxima de 87 anos. Os sinais e sintomas mais frequentes de disfagia orofaríngea foram presença de tosse e engasgo, além de outros relevantes, como pressão de língua diminuída, voz molhada, perda de peso e deglutição lenta. Conclusão: de acordo com os estudos revisados, os sinais e sintomas mais frequentes relacionados à disfagia orofaríngea nos idosos institucionalizados foram presença de tosse e engasgo, antes, durante ou após a deglutição.

Palavras-chave: Instituição de Longa Permanência para Idosos; Sinais e sintomas; Transtornos de Deglutição; Idoso; Revisão

\begin{abstract}
Purpose: To identify the most prevalent signs and symptoms of oropharyngeal dysphagia in elderly adults who live in old folks' home. Research strategy: Integrative review carried out in four databases: Embase, Lilacs, MEDLINE/Pubmed, and Web of Science using English terms and filters for language and age. Selection criteria: Studies available in the full-text form in English, Portuguese or Spanish, with no publication time restrictions, related to elderly people living in care homes who reported oropharyngeal dysphagia. Studies related to elderly people in the community or in hospitals and with other health issuesthat were not related to swallowing disorders were excluded. Results: Of 389 studies, 16 were included in this review, published between 1986 and 2020. There was a predominance of female participants whose minimum mean age was 71 and maximum, 87 . The most frequent signs and symptoms of oropharyngeal dysphagia were the presence of coughing and choking, in addition to other relevant ones, such as diminished tongue pressure, wet voice, weight loss, and slow swallowing. Conclusion: According to the reviewed studies, the most frequent signs and symptoms related to oropharyngeal dysphagia in elderly people living in care homes were (the) presence of coughing and choking, before, during or after swallowing.
\end{abstract}

Keywords: Homes for the Aged; Signs and symptoms; Deglutition Disorders; Elderly; Review

\footnotetext{
Trabalho realizado na Universidade Federal do Rio Grande do Norte - UFRN - Natal (RN), Brasil.

${ }^{1}$ Departamento de Fonoaudiologia, Universidade Federal do Rio Grande do Norte - UFRN - Natal (RN), Brasil.

${ }^{2}$ Departamento de Morfologia, Universidade Federal do Rio Grande do Norte - UFRN - Natal (RN), Brasil.

${ }^{3}$ Departamento de Cirurgia, Universidade Federal do Rio Grande do Norte - UFRN - Natal (RN), Brasil.

Conflito de interesses: Não.
}

Contribuição dos autores: SMLC foi responsável pela concepção e delineamento do estudo, coleta e análise dos dados, discussão dos resultados e conclusões e redação do manuscrito; DRPT colaborou no delineamento do estudo, coleta e análise dos dados e apresentação dos resultados; RVAC contribuiu no suporte teórico científico na metodologia do estudo, análise dos resultados e revisão do manuscrito; KVMT contribuiu no suporte teórico científico na metodologia do estudo, análise dos resultados e revisão do manuscrito; LMBMF contribuiu no suporte teórico científico na metodologia do estudo, análise dos resultados e revisão do manuscrito; HVMJ foi responsável pela concepção do estudo, análise metodológica, administração para execução da pesquisa, orientação e suporte na redação, revisão e edição do manuscrito.

Financiamento: O presente trabalho foi realizado com apoio da Coordenação de Aperfeiçoamento de Pessoal de Nível Superior - Brasil (CAPES) - Código de Financiamento 001.

Autor correspondente: Hipólito Virgilio Magalhães Junior. E-mail: h.vmagalhaes@gmail.com

Recebido: Março 19, 2021; Aceito: Dezembro 16, 2021 


\section{INTRODUÇÃO}

Com o aumento da longevidade, a população está se tornando cada vez mais envelhecida, o que requer atenção aos cuidados e acolhimento às necessidades do idoso, sendo a família a principal responsável por desempenhar a assistência às dificuldades que essa população demanda. Porém, com as mudanças da rotina familiar, isso vem sendo transformado ${ }^{(1)} \mathrm{e}$ uma das alternativas encontradas pelos familiares, ou pelo próprio idoso, é a procura por Instituições de Longa Permanência para Idosos (ILPIs), sejam elas públicas ou privadas ${ }^{(2)}$, para favorecer o cuidado de suas condições de saúde e socioeconômicas, além do amparo às circunstâncias externas, em decorrência das situações de solidão e medo da violência urbana ${ }^{(3)}$.

As ILPIs são instituições governamentais ou não governamentais, com caráter residencial coletivo, destinadas a pessoas com 60 anos ou mais, com ou sem suporte familiar, em condição de liberdade, dignidade e cidadania ${ }^{(4)}$. Elas atendem tanto idosos independentes, quanto aqueles dependentes de auxílio nas atividades de vida diárias ${ }^{(2)}$.

O idoso, quando reside em uma instituição, pode desenvolver problemas durante a alimentação, provocados, muitas vezes, pelo seu comportamento frente à alimentação, uso de consistências alimentares inadequadas impostas pela ILPI, dificuldades na postura e posicionamento para se alimentar, ou decorrentes do modo como o cuidador oferta o alimento, presença de alterações dentárias e recusa alimentar ${ }^{(5)}$.

Além disso, os idosos institucionalizados geralmente são mais fragilizados e, por isso, é comum não conseguirem compensar as modificações inerentes do processo de envelhecimento, em que há um conjunto de eventos biológicos que modificam as estruturas e funções estomatognáticas ${ }^{(6)}$, as quais, se acompanhadas de agravos à saúde associados à alimentação, a deglutição pode não ser compensada e, assim, surgir um quadro disfágico ${ }^{(7)}$.

No idoso, a disfagia, além de poder comprometer as fases antecipatória e esofágica, quando se faz um recorte para os desfechos voltados para a disfagia orofaríngea (DO), esta se apresenta como um transtorno da deglutição nas fases preparatória oral, oral propriamente dita e faríngea, caracterizado por um conjunto de sinais e sintomas que comprometem a eficiência e segurança em levar o alimento da cavidade oral ao esôfago ${ }^{(8)}$. Inclui, principalmente, dificuldade em mastigar o alimento e gerenciamento do bolo, escape e resíduos orais após comer, tosse, engasgo, voz molhada, pigarro frequente, necessidade de engolir várias vezes o alimento e dor ao engolir, tempo maior para completar uma refeição, postura incomum de cabeça ou pescoço ao engolir e perda de peso ${ }^{(9,10)}$.

Esse distúrbio pode ser potencializado quando o idoso reside em uma ILPI, em que os sinais e sintomas podem estar sendo negligenciados pela instituição e/ou pelo próprio idoso. Isso pode, ainda, trazer riscos de desnutrição, desidratação e aspiração, gerando pneumonias, fator que contribui para o aumento dos índices de mortalidade do idoso ${ }^{(11)}$. Também pode trazer impactos significativos na qualidade de vida, sono, desejo de se alimentar, interação social e em sua saúde mental ${ }^{(12)}$.

Assim, evidencia-se a importância de identificar precocemente os sinais e sintomas relacionados às alterações na deglutição nessa população, que podem apontar a importância de se sistematizar o rastreamento de disfagia orofaríngea como procedimento padrão a ser realizado por qualquer profissional de saúde devidamente calibrado, a fim de identificar idosos institucionalizados com possíveis alterações na eficiência e/ou segurança da deglutição, bem como a implementação de protocolos de acompanhamento da evolução dos quadros disfágicos rastreados, que devem ser confirmados por meio da avaliação fonoaudiológica para propiciar o estabelecimento de condutas de gerenciamento e intervenção da disfagia, dentre outras.

\section{OBJETIVO}

O objetivo desta revisão de literatura foi identificar os sinais e sintomas de disfagia orofaríngea mais presentes nos idosos que residem em Instituições de Longa Permanência.

\section{Estratégia de pesquisa}

Realizou-se uma revisão integrativa de acordo com as seguintes etapas: elaboração da pergunta norteadora; definição dos critérios de inclusão e exclusão; busca em base de dados com o uso de palavras-chave; seleção dos estudos; extração dos dados relevantes, como objetivo, metodologia, tamanho da amostra e principais resultados ${ }^{(13)}$.

Para o embasamento da pesquisa, foi desenvolvida a seguinte pergunta norteadora: "Quais os sinais e sintomas de disfagia orofaríngea em idosos institucionalizados?". Com isso, realizou-se um levantamento da literatura para selecionar estudos que respondessem a essa questão, utilizando as bases de dados Embase, Latin American and Caribbean Literature in Health Sciences (LILACS), MEDLINE/PubMed e Web of Science (Quadro 1). Para a realização da busca, foram utilizados termos na língua inglesa selecionados a partir da busca nos descritores MeSH (Medical Subject Headings) da PubMed, algoritmos da Embase e dos Descritores da saúde ("Deglutition Disorders"[MeSH], "Nursing Homes"[MeSH], "Homes for the Aged"[MeSH], Dysphagia, Institutionalized elderly) e aplicados filtros para idioma (inglês, português e espanhol) e idade (a partir de 60 anos), sem restrição de tempo de publicação.

Quadro 1. Estratégia de busca nas bases de dados

\begin{tabular}{|ll|}
\hline \multicolumn{1}{|c|}{ BASE } & \multicolumn{1}{c|}{ ESTRATÉGIA DE BUSCA } \\
\hline Embase & dysphagia:ti,ab,kw AND ('institutionalized elderly':ti,ab,kw OR 'nursing home':ti,ab,kw OR "home for the aged':ti,ab,kw) \\
& AND ([english]/lim OR [portuguese]/lim OR [spanish]/lim) AND [aged]/lim \\
LILACS & "DEGLUTITION DISORDER" [Palavras] and "HOMES FOR THE AGED” [Palavras] \\
MEDLINE/PubMed & "Deglutition Disorders"[Mesh] AND (“Nursing Homes"[Mesh] OR "Homes for the Aged"[Mesh]) \\
Web of Science & TÓPICO: (dysphagia) AND TÓPICO: ("nursing homes" OR "institutionalized elderly" OR "homes for the aged")
\end{tabular}




\section{Critérios de seleção}

Os critérios de inclusão foram: estudos originais com disponibilidade eletrônica na forma de texto completo, publicados em todo o período, nos idiomas inglês, português ou espanhol, realizados com população de idosos residentes em Instituições de Longa Permanência, que respondessem à questão norteadora e relatassem medidas relacionadas à disfagia orofaríngea. Foram excluídos resumos de congresso, pesquisas relacionadas a idosos residentes na comunidade ou que estivessem em ambiente hospitalar e/ou que abordassem outras condições de saúde que não se referiam aos distúrbios na deglutição.

\section{Análise dos dados}

A análise dos artigos encontrados foi dividida em três etapas. Inicialmente, foi realizada a análise quantitativa dos estudos encontrados, a partir das estratégias de busca, em que foi utilizado o software Mendeley para coletar e remover as referências duplicadas ${ }^{(14)}$. Posteriormente, todos os estudos foram inseridos no Rayyan ${ }^{(15)}$, que é um aplicativo de triagem inicial para análise de títulos e resumos dos estudos a serem escolhidos para a próxima fase, seguindo os critérios de elegibilidade. Na terceira etapa, todos os artigos selecionados foram lidos na íntegra e analisados os respectivos tópicos de introdução, metodologia, resultados e discussão, aplicando os critérios de elegibilidade para seleção daqueles que entrariam para esta revisão. A segunda e terceira etapas foram realizadas por dois avaliadores independentes e, após a leitura, realizou-se uma reunião de consenso, na qual, caso houvesse conflito nas decisões, um terceiro revisor tomaria a decisão final.

Os estudos incluídos foram avaliados quanto ao risco de viés por meio do Instrumento de Meta-Análise de Avaliação e Revisão Estatística (MASTARI) para Estudos Observacionais do Joanna Briggs Institute $(\mathrm{JBI})^{(16)}$. Dois revisores realizaram a avaliação do risco de viés separadamente e julgaram os artigos incluídos, marcando cada critério de avaliação com "sim", "não", "incerto" e "não aplicável". O risco de viés foi classificado como alto quando o estudo apresentou $49 \%$ dos critérios analisados como "sim"; moderado, quando atingiu $50 \%$ a $69 \%$ "sim" e baixo, quando foi identificado mais de $70 \%$ "sim". Quando necessário, as discordâncias foram resolvidas por meio da discussão com o terceiro revisor.

Após as três etapas, foram realizadas a organização e a sumarização dos estudos incluídos na revisão em um quadro com informações concisas, relacionadas a autores dos estudos, ano de publicação, país de origem, características da amostra referentes ao número de participantes, distribuição por sexo e média de idade; objetivo, tipo de estudo e nível de evidência ${ }^{(17)}$, método e principais resultados.

\section{RESULTADOS}

Foram encontrados 389 resultados, sendo que a maior parte dos estudos foi encontrada na base de dados Embase ( $\mathrm{n}=157)$, seguida pela MEDLINE/PubMed ( $\mathrm{n}=124)$ e Web of Science $(\mathrm{n}=104)$, e, em menor número, na LILACS $(\mathrm{n}=4)$. Após a remoção dos duplicados, 304 estudos foram selecionados para a leitura de título e resumo.
Ao final de todas as etapas do processo de construção desta revisão integrativa, foram incluídos 16 estudos, seguindo os critérios de elegibilidade (Figura 1).

Os estudos incluídos foram na língua inglesa e apenas 1 em português ${ }^{(18)}$. De todas as publicações, apenas 2 foram de estudos brasileiros, um da Região Sul ${ }^{(19)}$ e outro do Nordeste ${ }^{(18)}$. Em relação aos anos de publicação, variou de $1986^{(20)}$ a $2020^{(21)}$. Quanto à população estudada, houve predomínio de participantes idosos do sexo feminino, com média mínima de idade de 71 anos e máxima de 87 anos. $\mathrm{O}$ tamanho da amostra nos estudos variou desde um pequeno número de participantes $(\mathrm{n}=12)^{(22)}$ a uma grande quantidade de participantes $(\mathrm{n}=6.349)^{(23)}$. No que diz respeito às características das ILPIs, algumas eram subdivididas de acordo com o nível de cuidado e/ou a condição de saúde que o idoso apresentava e outras eram residências exclusivas para mulheres.

Os sinais e sintomas de DO mais frequentes foram: tosse ${ }^{(18,20-31)}$ e engasgo ${ }^{(22-24,26-28,30)}$ antes, durante ou após a deglutição. Outros sinais e sintomas, como pressão de língua diminuída ${ }^{(21,27,31,32)}$, voZ molhada $^{(18,20,28,29)}$, perda de peso ${ }^{(24,25,29,33)}$, deglutição lenta ${ }^{(20,24,29,30)}$, demora para completar as refeições ${ }^{(18,25,30,32)}$, escoamento anterior de saliva ${ }^{(20,29,30)}$, dificuldade na mastigação ${ }^{(20,24,29)}$, cuspir o alimento $^{(20,25,30)}$, redução da ingestão diária de alimentos ${ }^{(18,32)}$, beber líquido durante a refeição ${ }^{(24,28)}$ e presença de resíduos alimentares após a deglutição ${ }^{(18,30)}$ também foram encontrados.

Os sintomas menos frequentes foram: xerostomia ${ }^{(19)}$, desconforto na garganta ${ }^{(24)}$, comida grudando na garganta ${ }^{(24)}$, sensação de desconforto durante a ingestão de alimentos sólidos ${ }^{(24)}$, disfunção de lábio e língua, regurgitação nasal ${ }^{(20)} \mathrm{e}$ escape oral posterior de alimento $^{(20)}$.

O Quadro 2, apresenta a descrição dos artigos incluídos, com base no detalhamento das principais informações em relação ao tema, relacionadas aos objetivos, metodologia empregada e principais resultados em relação aos sinais e sintomas de disfagia orofaríngea (DO).

No que diz respeito ao risco de viés, 8 estudos foram julgados como alto risco ${ }^{(18,19,21-25,28)}, 4$ como moderado risco $^{(20,26,32,33)} \mathrm{e}$ 4 como baixo risco ${ }^{(27,29-31)}$. As limitações metodológicas em todos os estudos incluídos nesta revisão estavam relacionadas aos relatos deficientes para critérios de inclusão e exclusão da amostra, descrição dos sujeitos do estudo, fatores de confusão e estratégia de controle desses fatores. A maior parte dos estudos foi classificada como de baixo risco de viés no item relacionado à medida dos resultados de forma confiável e análise estatística apropriada. As Tabelas 1 e 2 resumem as avaliações obtidas por meio da ferramenta MASTARI da JBI ${ }^{(17)}$.

\section{DISCUSSÃO}

Nos estudos selecionados, observou-se que houve o predomínio de idosos do sexo feminino que residem em ILPI, tendência presente nos estudos com os idosos, em que há maior participação de pessoas do sexo feminino, em relação ao masculino e com média de idade acima de 70 anos ${ }^{(34,35)}$.

De maneira geral, um grande número de idosos apresenta queixas frequentes de $\mathrm{DO}$, principalmente referentes à tosse $\mathrm{e}$ engasgo antes, durante ou depois das refeições, sintomas que pressupõem uma alteração entre as fases oral e faríngea da deglutição ${ }^{(29,36)}$ e que podem sinalizar dificuldades na habilidade de deglutir de forma segura, em consequência do declínio cognitivo ou do comprometimento da função motora oral, ocasionada por 


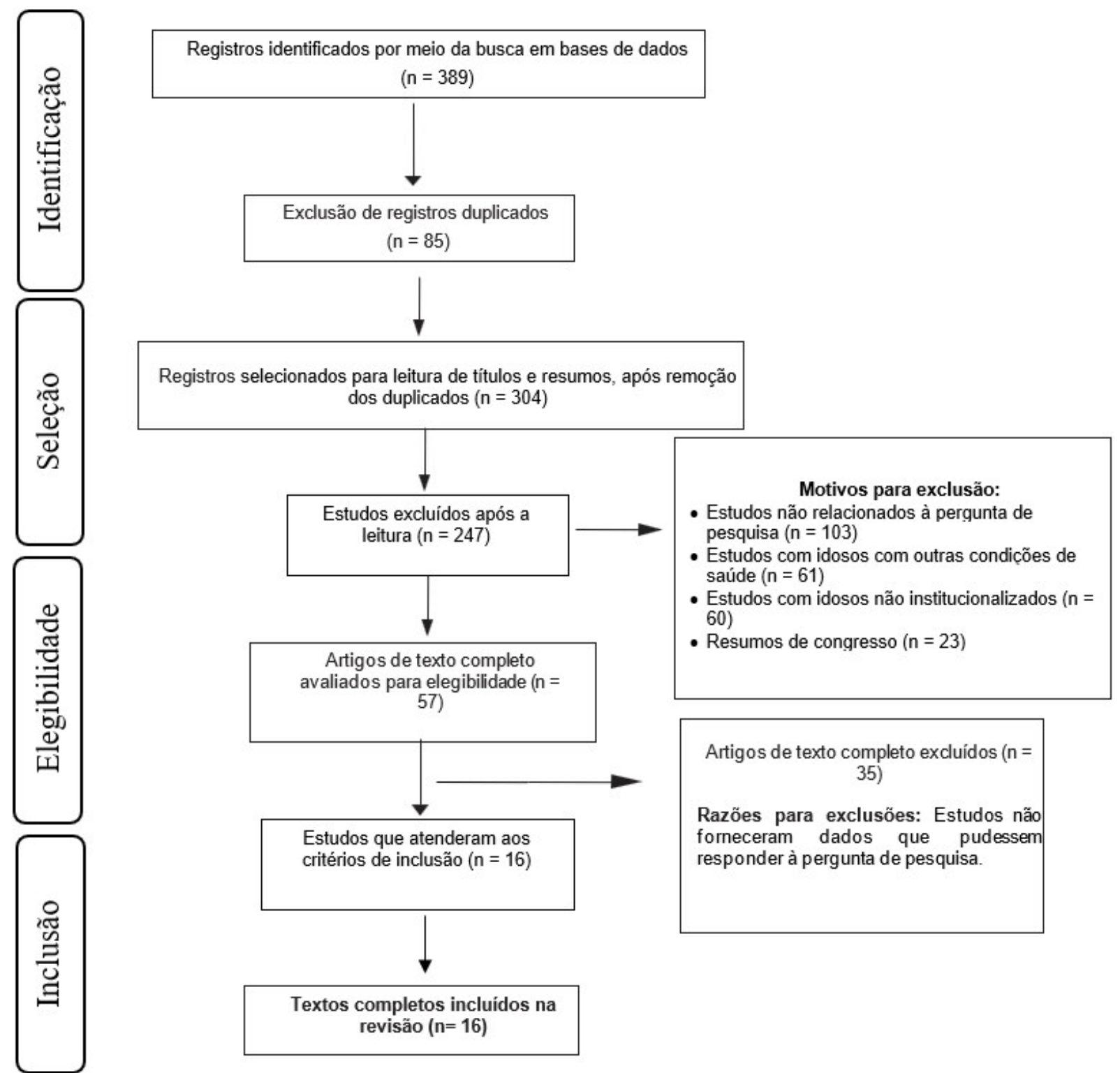

Figura 1. Fluxograma das etapas do processo de revisão integrativa Legenda: $\mathrm{n}=$ número de estudos

acidente vascular encefálico ou doenças neurodegenerativas, que são indicadores significativos associados à DO em idosos institucionalizados ${ }^{(11,20,28,37)}$.

Quando a dificuldade em deglutir está na fase faríngea, o idoso engasga, tosse e também pode broncoaspirar durante ou após a deglutição, e desenvolver um quadro de pneumonia ${ }^{(38)}$. A tosse é um sinal clássico de presença de penetração/aspiração relacionada à disfagia orofaríngea, sendo indicador da existência de sensibilidade na região laríngea, que estimula o ato reflexo de proteção das vias áreas ${ }^{(39)}$. Além disso, muitos idosos nas ILPIs frequentemente fazem suas refeições na cama, o que contribui para o desencadeamento de tosse e engasgos e causar asfixia e aspiração ${ }^{(25)}$.

A percepção do engasgo é comum entre a população idosa institucionalizada e a principal visão deles em relação às causas desse sintoma é o envelhecimento ${ }^{(40,41)}$, associado à presença de sentimentos/sensações negativas, ligando-os à morte, à falta de ar, ao medo ou à negação do sintoma ${ }^{(40)}$.

É relevante observar que, no idoso, a presença de voz molhada pode estar relacionada ao risco de penetração, como um sinal indicativo de estase de secreções, líquidos ou alimentos no vestíbulo laríngeo, bem como ao risco de aspiração ${ }^{(18,42)}$. Nos indivíduos mais velhos, o início dos eventos faríngeos e laríngeos, incluindo o fechamento das vias aéreas, é significativamente mais demorado do que em adultos ${ }^{(43)} \mathrm{e}$, quando esse mecanismo de deglutição está funcionalmente alterado, o risco de ocorrer penetração nas vias aéreas pode ser ainda maior ${ }^{(44)}$.

A deglutição lenta pode ser um indicador de transtorno em iniciar a transferência do bolo para a região faríngea, possivelmente decorrente do envelhecimento, que interfere no início da reação faríngea ${ }^{(43)}$ e na eficiência da mastigação, que pode estar significativamente mais atrasada nos idosos, 
Quadro 2. Caracterização dos estudos incluídos na revisão integrativa

\begin{tabular}{|c|c|c|c|c|c|}
\hline Autor, ano, país & Amostra & Objetivos & Método & $\begin{array}{l}\text { Principais } \\
\text { resultados }\end{array}$ & TE/NE \\
\hline $\begin{array}{l}\text { Brochier et al. (2018), } \\
\text { Brasil(19) }^{(19)}\end{array}$ & $\begin{array}{l}115 \text { em três ILPI, } 67 \% \\
\text { do sexo feminino, com } \\
\text { idade superior a } 81 \\
\text { anos. }\end{array}$ & $\begin{array}{l}\text { Avaliar a associação } \\
\text { das variáveis } \\
\text { sociodemográficas, } \\
\text { comportamentais } \\
\text { e das condições } \\
\text { bucais com a } \\
\text { presença de disfagia } \\
\text { orofaríngea em idosos } \\
\text { institucionalizados. }\end{array}$ & $\begin{array}{l}\text { Foi realizada } \\
\text { avaliação sensório- } \\
\text { motora oral e } \\
\text { diagnóstico } \\
\text { clínico de disfagia } \\
\text { orofaríngea com } \\
\text { teste de deglutição } \\
\text { indireta, avaliação } \\
\text { oral e inventário de } \\
\text { xerostomia }(\mathrm{XI}) \text {. }\end{array}$ & $\begin{array}{l}\text { A xerostomia } \\
\text { foi o único sinal } \\
\text { que apresentou } \\
\text { associação positiva } \\
\text { com disfagia } \\
\text { orofaríngea. } \\
(p<0,001) .\end{array}$ & $\begin{array}{l}\text { Transversal } \\
\text { Nível VI }\end{array}$ \\
\hline $\begin{array}{l}\text { Ekberg et al. (2002), } \\
\text { Alemanha, França, } \\
\text { Espanha e Reino } \\
\text { Unido } \\
\text { (24) }\end{array}$ & $\begin{array}{l}360 \text { em } 37 \text { ILPI, } 67 \% \\
\text { do sexo feminino, com } \\
\text { média de idade de } \\
71,6 \text { anos. }\end{array}$ & $\begin{array}{l}\text { Determinar os } \\
\text { efeitos da disfagia } \\
\text { em aspectos sociais } \\
\text { e psicológicos } \\
\text { relacionados à } \\
\text { qualidade de vida e } \\
\text { investigar a relação } \\
\text { entre a condição e } \\
\text { seu diagnóstico e } \\
\text { tratamento. }\end{array}$ & $\begin{array}{l}\text { A avaliação se } \\
\text { deu por meio de } \\
\text { entrevistas usando } \\
\text { a revisão modificada } \\
\text { do questionário de } \\
\text { Gustafsson e Tibbling, } \\
\text { para eliciar problemas } \\
\text { relacionados à } \\
\text { disfagia (informações } \\
\text { básicas, hábitos } \\
\text { alimentares, } \\
\text { sentimentos pessoais, } \\
\text { busca de ajuda e } \\
\text { situação médica). }\end{array}$ & $\begin{array}{l}\text { Foram encontrados } \\
\text { sintomas de comida } \\
\text { grudando na garganta } \\
\text { ( } 55 \%) \text {, desconforto } \\
\text { ou dor de garganta } \\
(41 \%) \text {, engasgo } \\
\text { e tosse (46\%) e } \\
\text { desconforto ao comer } \\
\text { alimentos sólidos } \\
\text { ( } 55 \%) \text {, mastigação e } \\
\text { deglutição mais lentas } \\
\text { (32\%), hábito de } \\
\text { beber líquido durante } \\
\text { a refeição (27\%) e } \\
\text { perda de peso (44\%). }\end{array}$ & $\begin{array}{l}\text { Transversal } \\
\text { Nível VI }\end{array}$ \\
\hline $\begin{array}{l}\text { Gilmore- } \\
\text { Bykovskyi et al. } \\
\text { (2018), Estados } \\
\text { Unidos }^{(22)}\end{array}$ & $\begin{array}{l}12 \text { em uma ILPI para } \\
\text { demência, } 50 \% \text { do } \\
\text { sexo feminino, com } \\
\text { média de idade de } 84 \\
\text { anos. }\end{array}$ & $\begin{array}{l}\text { Identificar } \\
\text { associações } \\
\text { temporais entre } \\
\text { a abordagem do } \\
\text { cuidador, sintomas } \\
\text { comportamentais e } \\
\text { eventos de aspiração } \\
\text { entre residentes de } \\
\text { ILPI, com demência. }\end{array}$ & $\begin{array}{l}\text { Foram avaliados } \\
\text { por meio de vídeo- } \\
\text { observação de duas } \\
\text { refeições relacionadas } \\
\text { às ações do cuidador } \\
\text { centradas na } \\
\text { pessoa (residente), } \\
\text { centradas nas } \\
\text { tarefas do cuidador } \\
\text { e indicadores de } \\
\text { aspiração definidos, } \\
\text { como tosse ou } \\
\text { engasgo durante ou } \\
\text { após a deglutição. }\end{array}$ & $\begin{array}{l}\text { Identificados sinais } \\
\text { de tosse ou engasgo } \\
\text { durante as refeições, } \\
\text { que foram indicadores } \\
\text { observáveis de } \\
\text { eventos de aspiração } \\
\text { laringotraqueal, } \\
\text { associados às ações } \\
\text { centradas nas tarefas } \\
\text { do cuidador }(96 \%) \text {. }\end{array}$ & $\begin{array}{l}\text { Transversal } \\
\text { Nível VI }\end{array}$ \\
\hline $\begin{array}{l}\text { Huppertz et al. (2018), } \\
\text { Holanda }^{(23)}\end{array}$ & $\begin{array}{l}6.349 \text { em } 65 \text { ILPI, } \\
70,2 \% \text { do sexo } \\
\text { feminino, com média } \\
\text { de idade de } 84,5 \\
\text { anos. }\end{array}$ & $\begin{array}{l}\text { Avaliar a associação } \\
\text { entre disfagia } \\
\text { orofaríngea e } \\
\text { desnutrição em } \\
\text { residentes de lares de } \\
\text { idosos holandeses. }\end{array}$ & $\begin{array}{l}\text { Foi aplicado um } \\
\text { questionário } \\
\text { estabelecido com } \\
\text { base na literatura } \\
\text { e consulta de } \\
\text { especialistas, que } \\
\text { incluiu questões sobre } \\
\text { sintomas relevantes } \\
\text { para detectar DO: } \\
\text { tem problemas para } \\
\text { engolir e espirra ou } \\
\text { tosse ao engolir? } \\
\text { A desnutrição foi } \\
\text { indicada com base } \\
\text { no Índice de Massa } \\
\text { Corporal (IMC). }\end{array}$ & $\begin{array}{l}\text { Foi observado que } \\
\text { quase metade dos } \\
\text { que tinham disfagia } \\
\text { indicou sinais de } \\
\text { tosse no ato de } \\
\text { deglutir }(46,9 \%) \text { e } \\
\text { quase todos esses } \\
\text { residentes que } \\
\text { tossiam apresentaram } \\
\text { problemas gerais para } \\
\text { engolir }(82,2 \%) \text {. }\end{array}$ & Transversal Nível VI \\
\hline $\begin{array}{l}\text { Jukic Peladic et al. } \\
(2018) \text {, Itália(33) }\end{array}$ & $\begin{array}{l}1.299 \text { durante } 6 \\
\text { meses e } 971 \text { por } 12 \\
\text { meses, } 71,5 \% \text { do } \\
\text { sexo feminino, com } \\
\text { média de idade de } \\
83,5 \text { anos. }\end{array}$ & $\begin{array}{l}\text { Estimar prevalência } \\
\text { de disfagia e fatores } \\
\text { associados e } \\
\text { investigar a influência } \\
\text { da disfagia e das } \\
\text { terapias nutricionais } \\
\text { realizadas em } \\
\text { indivíduos disfágicos } \\
\text { nos desfechos } \\
\text { clínicos. }\end{array}$ & $\begin{array}{l}\text { Avaliação clínica } \\
\text { com a coleta de } \\
\text { informações sobre } \\
\text { problemas de } \\
\text { deglutição e revisão } \\
\text { do histórico médico. O } \\
\text { estado nutricional foi } \\
\text { avaliado por meio das } \\
\text { informações sobre } \\
\text { perda de peso. }\end{array}$ & $\begin{array}{l}\text { Os sujeitos com } \\
\text { disfagia apresentaram } \\
\text { sinais de maior } \\
\text { perda de peso, } \\
\text { em comparação } \\
\text { aos indivíduos não } \\
\text { disfágicos (14,6\%, } \\
\text { p<0,001). }\end{array}$ & $\begin{array}{l}\text { Coorte prospectivo } \\
\text { Nível IV }\end{array}$ \\
\hline
\end{tabular}

Legenda: ILPI = Instituição de Longa Permanência para Idosos; DO = disfagia orofaríngea; \% = percentual; TE = Tipo de estudo; NE = Nível de evidência, $\mathrm{n}$ = número de idosos 
Quadro 2. Continuação...

\begin{tabular}{|c|c|c|c|c|c|}
\hline Autor, ano, país & Amostra & Objetivos & Método & $\begin{array}{l}\text { Principais } \\
\text { resultados }\end{array}$ & TE/NE \\
\hline $\begin{array}{l}\text { Kayser-Jones e } \\
\text { Pengilly (1999), } \\
\text { Estados Unidos }\end{array}$ & $\begin{array}{l}82 \text { em duas ILPI, sem } \\
\text { dados de sexo, com } \\
\text { média de idade de } \\
83,4 \text { anos. }\end{array}$ & $\begin{array}{l}\text { Identificar os fatores } \\
\text { que influenciam a } \\
\text { ingestão nutricional } \\
\text { em lares de idosos. }\end{array}$ & $\begin{array}{l}\text { Foram realizadas } \\
\text { observações por } \\
\text { cerca de } 6 \text { meses, } \\
\text { de todas as três } \\
\text { refeições. Cada } \\
\text { residente passou } \\
\text { por uma triagem } \\
\text { de disfagia à beira } \\
\text { do leito, por um } \\
\text { fonoaudiólogo. }\end{array}$ & $\begin{array}{l}\text { Foram observados } \\
\text { e relatados pelas } \\
\text { residentes sinais } \\
\text { de demora para } \\
\text { completar a fase } \\
\text { oral, perda de peso, } \\
\text { idosos que cuspiam } \\
\text { a comida, tosse } \\
\text { frequente ao comer e } \\
\text { beber líquidos. }\end{array}$ & $\begin{array}{l}\text { Transversal } \\
\text { Nível VI }\end{array}$ \\
\hline $\begin{array}{l}\text { Lin et al. (2002) } \\
\text { Taiwan } \\
(26)\end{array}$ & $\begin{array}{l}1.221 \text { em } 18 \text { ILPI, } \\
48,1 \% \text { do sexo } \\
\text { feminino, com média } \\
\text { de idade de } 77,07 \\
\text { anos. }\end{array}$ & $\begin{array}{l}\text { Investigar a } \\
\text { prevalência } \\
\text { da deglutição } \\
\text { prejudicada em } \\
\text { residentes de } \\
\text { instituições de longa } \\
\text { permanência em } \\
\text { Taiwan. }\end{array}$ & $\begin{array}{l}\text { Avaliação foi baseada } \\
\text { em questionários } \\
\text { de autorrelato de } \\
\text { dificuldade na } \\
\text { deglutição e teste } \\
\text { de deglutição } \\
\text { cronometrada de } \\
\text { líquido. }\end{array}$ & $\begin{array}{l}\text { Os indivíduos } \\
\text { pontuaram para } \\
\text { sinais e sintomas } \\
\text { de dificuldade para } \\
\text { engolir e foi verificada } \\
\text { a ocorrência de tosse } \\
\text { e engasgo durante o } \\
\text { teste de deglutição } \\
\text { cronometrada. }\end{array}$ & $\begin{array}{l}\text { Transversal } \\
\text { Nível VI }\end{array}$ \\
\hline $\begin{array}{l}\text { Namasivayam et al. } \\
\text { (2016), Canadá(32) }\end{array}$ & $\begin{array}{l}20 \text { em uma ILPI, } \\
12,60 \% \text { do sexo } \\
\text { feminino, com média } \\
\text { de idade de } 85 \text { anos. }\end{array}$ & $\begin{array}{l}\text { Explorar a força } \\
\text { da língua e sua } \\
\text { associação com } \\
\text { sinais de deficiência } \\
\text { na deglutição, com } \\
\text { base no rastreamento } \\
\text { de disfagia e nas } \\
\text { observações } \\
\text { durante as refeições, } \\
\text { tempo para comer } \\
\text { e quantidade } \\
\text { de alimentos } \\
\text { consumidos. }\end{array}$ & $\begin{array}{l}\text { Foi usada a versão } \\
\text { modificada da } \\
\text { triagem para disfagia } \\
\text { neurológica aguda } \\
\text { (STAND), lowa } \\
\text { Oral Performance } \\
\text { Instrument (IOPI) } \\
\text { para medir as } \\
\text { pressões máxima da } \\
\text { língua e observadas } \\
\text { as refeições para } \\
\text { determinar a ingestão } \\
\text { e duração alimentar. }\end{array}$ & $\begin{array}{l}\text { Foi identificado } \\
\text { que os que tinham } \\
\text { força máxima da } \\
\text { língua reduzida } \\
\text { apresentaram sinais } \\
\text { de dificuldade para } \\
\text { engolir durante as } \\
\text { refeições, levaram, } \\
\text { em média, vinte } \\
\text { minutos a mais } \\
\text { para completar as } \\
\text { refeições e tiveram } \\
\text { redução da ingestão } \\
\text { diária de alimentos ( } p \\
<0,05 \text { ). }\end{array}$ & $\begin{array}{l}\text { Transversal } \\
\text { Nível VI }\end{array}$ \\
\hline $\begin{array}{l}\text { Namasivayam- } \\
\text { MacDonald et al. } \\
\text { (2017) Canadá(27) }\end{array}$ & $\begin{array}{l}639 \text { em } 32 \text { ILPI, } \\
68,9 \% \text { do sexo } \\
\text { feminino, com média } \\
\text { de idade de } 87 \text { anos. }\end{array}$ & $\begin{array}{l}\text { Estudar as } \\
\text { associações entre } \\
\text { estado nutricional, } \\
\text { medidas de ingestão } \\
\text { de alimentos durante } \\
\text { as refeições, sinais } \\
\text { clínicos sugestivos de } \\
\text { disfagia e redução da } \\
\text { força da língua. }\end{array}$ & $\begin{array}{l}\text { Foi realizada } \\
\text { observação do } \\
\text { comportamento dos } \\
\text { idosos durante as } \\
\text { refeições, triagem } \\
\text { para disfagia } \\
\text { neurológica aguda } \\
\text { (STAND) e a } \\
\text { medida de força de } \\
\text { língua com o lowa } \\
\text { Oral Performance } \\
\text { Instrument (IOPI), em } \\
\text { um subconjunto de } 80 \\
\text { residentes. }\end{array}$ & $\begin{array}{l}\text { Foram registrados, } \\
\text { na maioria dos } \\
\text { residentes, sinais de } \\
\text { tosse e, em menor } \\
\text { número, engasgo. } \\
\text { Também foram } \\
\text { observados sinais de } \\
\text { menor pressão de } \\
\text { língua em idosos com } \\
\text { suspeita de disfagia } \\
(p<0,05) .\end{array}$ & Transversal Nível VI \\
\hline $\begin{array}{l}\text { Nogueira e Reis } \\
\text { (2013), Portugal(28) }\end{array}$ & $\begin{array}{l}266 \text { em oito ILPI, } 75 \% \\
\text { do sexo feminino, com } \\
\text { média de idade de } 82 \\
\text { anos. }\end{array}$ & $\begin{array}{l}\text { Determinar a } \\
\text { prevalência de } \\
\text { distúrbios de } \\
\text { deglutição em idosos } \\
\text { de ILPI e identificar } \\
\text { sua relação com } \\
\text { o desempenho } \\
\text { cognitivo e funcional, } \\
\text { e as variáveis que } \\
\text { explicam os distúrbios } \\
\text { da deglutição } \\
\text { autopercebidos. }\end{array}$ & $\begin{array}{l}\text { Os perfis de } \\
\text { deglutição e } \\
\text { alimentação foram } \\
\text { avaliados por meio } \\
\text { do teste } 3 \text { ounce } \\
\text { Water Swallow Test (3 } \\
\text { OZwst) e questionário } \\
\text { Dysphagia Self-Test } \\
\text { (DST) para os idosos } \\
\text { que conseguiram } \\
\text { responder. }\end{array}$ & $\begin{array}{l}\text { Foram registrados, } \\
\text { a partir do } 3 \text { OZwst, } \\
\text { sinais de tosse e } \\
\text { voz molhada, sendo } \\
\text { esta mais prevalente } \\
(10,3 \%) \text {. Os sintomas } \\
\text { mais frequentes } \\
\text { autorreferidos a } \\
\text { partir do DST foram } \\
\text { engasgar ou tossir } \\
\text { com alimentos sólidos } \\
\text { ou líquidos (49\%) e } \\
\text { necessidade de beber } \\
\text { algo após deglutir } \\
\text { (47\%). }\end{array}$ & Transversal Nível VI \\
\hline
\end{tabular}

Legenda: ILPI = Instituição de Longa Permanência para Idosos; DO = disfagia orofaríngea; \% = percentual; TE = Tipo de estudo; NE = Nível de evidência, $\mathrm{n}$ = número de idosos 
Quadro 2. Continuação...

\begin{tabular}{|c|c|c|c|c|c|}
\hline Autor, ano, país & Amostra & Objetivos & Método & $\begin{array}{l}\text { Principais } \\
\text { resultados }\end{array}$ & TE/NE \\
\hline $\begin{array}{l}\text { Nomura et al. (2020), } \\
\text { Japão(21) }\end{array}$ & $\begin{array}{l}69 \text { em uma ILPI, } \\
81,15 \% \text { do sexo } \\
\text { feminino, com média } \\
\text { de idade de } 86,23 \\
\text { anos. }\end{array}$ & $\begin{array}{l}\text { Analisar a relação } \\
\text { entre as funções orais } \\
\text { e a consistência do } \\
\text { alimento fornecido. }\end{array}$ & $\begin{array}{l}\text { Foram verificados } \\
\text { a umidade oral, } \\
\text { estado de higiene } \\
\text { oral, pressão oclusal } \\
\text { máxima e pressão } \\
\text { da língua pelo Dental } \\
\text { Prescale, função } \\
\text { da língua e lábios } \\
\text { por diadococinesia } \\
\text { oral, mastigação } \\
\text { com o sensor Gluco } \\
\text { GSIl e deglutição } \\
\text { por um questionário } \\
\text { relacionado aos } \\
\text { sintomas de DO. }\end{array}$ & $\begin{array}{l}\text { O sintoma de } \\
\text { despertar devido } \\
\text { à tosse durante o } \\
\text { sono apresentou } \\
\text { associação } \\
\text { relativamente alta } \\
\text { com dificuldade na } \\
\text { deglutição e muitos } \\
\text { desses idosos } \\
\text { apresentaram sinal de } \\
\text { disfunção de língua e } \\
\text { lábios }(p=0,027) \text {. }\end{array}$ & Transversal Nível VI \\
\hline $\begin{array}{l}\text { Park et al. (2013), } \\
\text { Coreia do Sul(29) }\end{array}$ & $\begin{array}{l}395 \text { em duas ILPI, } \\
76,5 \% \text { do sexo } \\
\text { feminino e } 76,7 \% \\
\text { tinham } 75 \text { anos ou } \\
\text { mais. }\end{array}$ & $\begin{array}{l}\text { Avaliar a prevalência } \\
\text { de disfagia em } \\
\text { residentes de lares } \\
\text { de idosos na Coreia } \\
\text { do Sul e identificar os } \\
\text { fatores associados à } \\
\text { disfagia. }\end{array}$ & $\begin{array}{l}\text { Foram coletadas } \\
\text { informações sobre } \\
\text { os sintomas de } \\
\text { alerta, sinais de } \\
\text { disfagia e problemas } \\
\text { de deglutição, } \\
\text { relacionados } \\
\text { à capacidade } \\
\text { de deglutição e } \\
\text { gravidade da disfagia } \\
\text { por meio do Gugging } \\
\text { Swallowing Screen } \\
\text { (GUSS). }\end{array}$ & $\begin{array}{l}\text { Entre os sinais } \\
\text { encontrados, voz } \\
\text { molhada }(14,4 \%) \text {, } \\
\text { escoamento de saliva } \\
(9,1 \%) \text {, deglutição } \\
\text { lenta }(58,2 \%) \text {, tosse } \\
(24 \%) \text {, dificuldade } \\
\text { na mastigação } \\
(63,5 \%) \text { e perda } \\
\text { de peso ( } 31,7 \%) \\
(,) \text { foram fatores de } \\
\text { risco associados } \\
\text { significativamente à } \\
\text { disfagia ( } p<0,001) .\end{array}$ & Transversal Nível VI \\
\hline $\begin{array}{l}\text { Roque et al. (2010), } \\
\text { Brasil(18) }\end{array}$ & $\begin{array}{l}30 \text { em uma ILPI, do } \\
\text { sexo feminino, com } \\
\text { média de idade de } \\
83,73 \text { anos. }\end{array}$ & $\begin{array}{l}\text { Descrever a dinâmica } \\
\text { da alimentação } \\
\text { de idosas } \\
\text { institucionalizadas, no } \\
\text { que diz respeito aos } \\
\text { aspectos clínicos da } \\
\text { deglutição, cognitivos, } \\
\text { comportamentais e } \\
\text { ambientais ligados à } \\
\text { alimentação. }\end{array}$ & $\begin{array}{l}\text { Foram realizados } \\
\text { a observação de } \\
\text { uma refeição em } \\
\text { tempo real e o } \\
\text { registro audiovisual } \\
\text { para verificar os } \\
\text { aspectos atitudinais e } \\
\text { comportamentais das } \\
\text { idosas, dependência } \\
\text { requerida e realização } \\
\text { da inspeção da } \\
\text { cavidade oral. }\end{array}$ & $\begin{array}{l}\text { Foi observado } \\
\text { que os sinais mais } \\
\text { presentes foram: } \\
\text { resíduos alimentares } \\
(23,3 \%) \text { e alteração } \\
\text { vocal }(16,7 \%) \text { após } \\
\text { a deglutição e tosse } \\
\text { (20\%) durante a } \\
\text { alimentação. Também } \\
\text { foi identificada } \\
\text { ingestão oral } \\
\text { pobre (26,7\%) e } \\
\text { tempo maior para } \\
\text { alimentação }(53,3 \%) \text {. }\end{array}$ & Transversal Nível VI \\
\hline $\begin{array}{l}\text { Siebens et al. (1986), } \\
\text { Estados Unidos }\end{array}$ & $\begin{array}{l}131 \text { em uma ILPI, } \\
79 \% \text { do sexo } \\
\text { feminino, com média } \\
\text { de idade de } 81,7 \\
\text { anos. }\end{array}$ & $\begin{array}{l}\text { Identificar os fatores } \\
\text { associados à perda } \\
\text { da capacidade } \\
\text { alimentar. }\end{array}$ & $\begin{array}{l}\text { Foi aplicado um } \\
\text { questionário } \\
\text { referente à dieta } \\
\text { alimentar, frequência } \\
\text { de alimentação } \\
\text { própria, disfunção } \\
\text { de membros } \\
\text { superiores e sinais } \\
\text { de disfagia. Também } \\
\text { foram avaliadas a } \\
\text { cognição, capacidade } \\
\text { de engolir líquidos } \\
\text { e semissólidos e } \\
\text { funções motoras } \\
\text { das estruturas } \\
\text { orofaríngeas. }\end{array}$ & $\begin{array}{l}\text { Idosos dependentes } \\
\text { exibiram maiores } \\
\text { sinais no estágio } \\
\text { oral, como cuspir o } \\
\text { alimento, engasgar-se } \\
\text { ( } n=28 \text { ), incapacidade } \\
\text { de mastigar ( } n=19) \text {, } \\
\text { escoar saliva ( } n=26) \\
\text { regurgitação nasal } \\
\text { ( } n=3 \text { ), escape oral } \\
\text { de alimento ( } n=13 \text { ) } \\
\text { e demora para } \\
\text { deglutir ( } n=27) \text {. Na } \\
\text { fase faríngea, foram } \\
\text { identificados sinais } \\
\text { de tosse ao deglutir } \\
\text { líquidos }(n=51) \text {, } \\
\text { engasgo durante as } \\
\text { refeições }(n=36) \text { e voz } \\
\text { úmida }(n=12) \text {. }\end{array}$ & Transversal Nível VI \\
\hline
\end{tabular}

Legenda: ILPI = Instituição de Longa Permanência para Idosos; DO = disfagia orofaríngea; \% = percentual; TE = Tipo de estudo; NE = Nível de evidência, $\mathrm{n}$ = número de idosos 
Quadro 2. Continuação...

\begin{tabular}{|c|c|c|c|c|c|}
\hline Autor, ano, país & Amostra & Objetivos & Método & $\begin{array}{c}\text { Principais } \\
\text { resultados }\end{array}$ & TE/NE \\
\hline $\begin{array}{l}\text { Steele et al. (1997) } \\
\text { Canadá(30) }\end{array}$ & $\begin{array}{l}372 \text { em uma ILPI, em } \\
\text { níveis de cuidado, } \\
280 \text { do sexo feminino, } \\
\text { com média de idade } \\
\text { de } 87 \text { anos. }\end{array}$ & $\begin{array}{l}\text { Prevalência de } \\
\text { dificuldades } \\
\text { identificáveis } \\
\text { relacionadas à } \\
\text { alimentação em } \\
\text { uma instituição de } \\
\text { multicuidados para } \\
\text { idosos e determinar } \\
\text { sua distribuição } \\
\text { em vários níveis de } \\
\text { atenção. }\end{array}$ & $\begin{array}{l}\text { Foi realizada a } \\
\text { observação de uma } \\
\text { única refeição e } \\
\text { anotadas a ocorrência } \\
\text { e frequência de } \\
\text { uma lista de } 12 \\
\text { problemas específicos } \\
\text { relacionados a comer/ } \\
\text { engolir ou quaisquer } \\
\text { dificuldades de } \\
\text { mastigação. }\end{array}$ & $\begin{array}{l}\text { Observado maior } \\
\text { prevalência de sinais } \\
\text { de demora para } \\
\text { completar a refeição } \\
\text { (29 minutos), tossir ou } \\
\text { se engasgar ( } 28 \%) \text {, } \\
\text { escoar saliva ( } 71 \%) \text {, } \\
\text { deglutição ausente ou } \\
\text { lenta (31\%), resíduos } \\
\text { orais e cuspir a } \\
\text { comida }(33 \%) \text {. }\end{array}$ & Transversal Nível VI \\
\hline $\begin{array}{l}\text { Yoshida et al. (2006) } \\
\text { Japão(31) }\end{array}$ & $\begin{array}{l}145 \text { em cinco ILPI, } \\
109 \text { do sexo feminino, } \\
\text { com média de idade } \\
\text { de } 83 \text { anos. }\end{array}$ & $\begin{array}{l}\text { Determinar a relação } \\
\text { entre a força da } \\
\text { língua e os sinais de } \\
\text { tosse e demonstrar } \\
\text { o valor clínico da } \\
\text { medida da pressão da } \\
\text { língua na avaliação } \\
\text { da deglutição. }\end{array}$ & $\begin{array}{l}\text { A identificação } \\
\text { dos problemas de } \\
\text { deglutição se deu } \\
\text { a partir do relato de } \\
\text { tosse nas refeições, } \\
\text { classificada de leve a } \\
\text { intensa e, para avaliar } \\
\text { a pressão da língua, } \\
\text { foi usado um protótipo } \\
\text { de um dispositivo de } \\
\text { registro de pressão } \\
\text { lingual. }\end{array}$ & $\begin{array}{l}\text { A maioria dos idosos } \\
\text { relatou sintomas de } \\
\text { tosse leve e aqueles } \\
\text { com problemas } \\
\text { de deglutição } \\
\text { definidos relataram } \\
\text { tosse intensa. } \\
\text { Foi identificada } \\
\text { menor pressão de } \\
\text { língua, que esteve } \\
\text { significativamente } \\
\text { relacionada à tosse } \\
\text { na hora das refeições } \\
(p<0,001) .\end{array}$ & Transversal Nível VI \\
\hline
\end{tabular}

Legenda: ILPI = Instituição de Longa Permanência para Idosos; DO = disfagia orofaríngea; \% = percentual; TE = Tipo de estudo; NE = Nível de evidência, $\mathrm{n}$ = número de idosos

Tabela 1. Avaliação dos riscos de viés dos estudos transversais

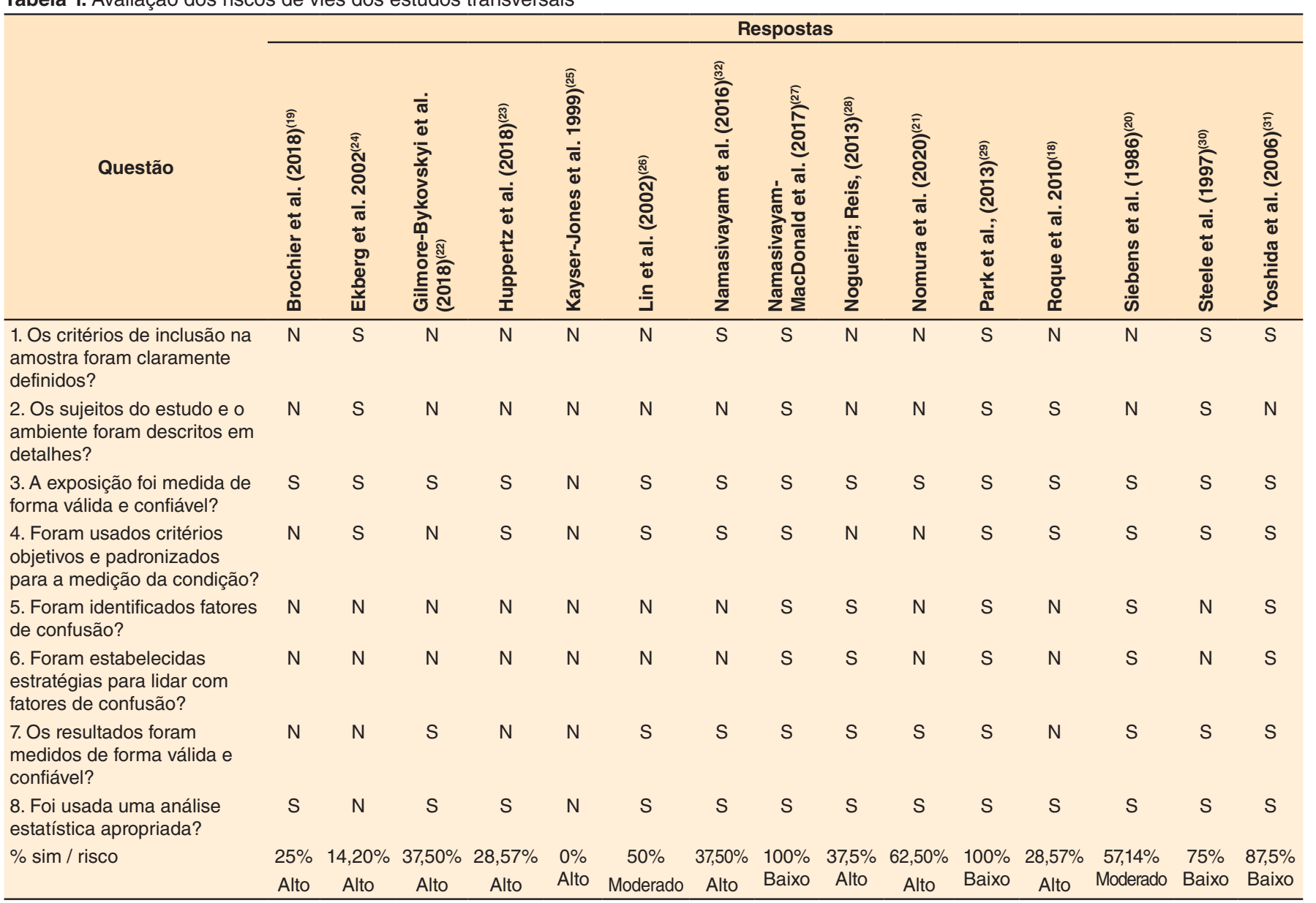

Legenda: $\mathrm{S}=\mathrm{Sim} ; \mathrm{N}=\mathrm{Não} ; \%$ = percentual 
Tabela 2. Avaliação dos riscos de viés do estudo de coorte

\section{Questão}

1. Os dois grupos eram semelhantes e recrutados na mesma população?

2. As exposições foram medidas de forma semelhante para designar as pessoas para grupos expostos e não expostos?

3. A exposição foi medida de forma válida e confiável?

4. Foram identificados fatores de confusão?

5. Foram estabelecidas estratégias para lidar com fatores de confusão?

6. Os grupos/participantes estavam livres do desfecho no início do estudo (ou no momento da exposição)?

7. Os resultados foram medidos de forma válida e confiável?

8. O tempo de acompanhamento foi relatado e suficiente para ser longo o suficiente para que os resultados ocorressem?

9. O acompanhamento foi completo e, em caso negativo, os motivos da perda de acompanhamento foram descritos e explorados?

10. Foram utilizadas estratégias para lidar com o acompanhamento incompleto?

11. Foi usada uma análise estatística apropriada?

$\% \operatorname{sim} /$ risco

Legenda: $\mathrm{S}=\operatorname{Sim} ; \mathrm{N}=\mathrm{Não} \%$ = percentual

quando comparada aos adultos com menos de $45 \operatorname{anos}^{(44)}$. Esse processo alterado pode gerar presença de resíduos na cavidade oral, que requerem a realização de deglutições múltiplas, por dificuldade na propulsão oral( ${ }^{(4)}$.

A baixa frequência de presença de regurgitação nasal de alimentos e líquidos justifica-se por ser considerada como um dos sintomas menos comumente percebidos pelos idosos ${ }^{(46)}$.

A literatura indica que o pico pressórico da língua diminuiu moderadamente com o aumento da idade, com observação de que aqueles classificados com pressão lingual extremamente fraca foram, significativamente, os mais velhos ${ }^{(46-48)}$. Também indivíduos com perda do suporte oclusal provocada por ausências dentárias possuíam instabilidade no padrão de movimentação de língua, o que pode contribuir para a menor média de pico pressórico e, como consequência, para deficiências na retenção e manipulação do bolo alimentar e na propulsão do bolo da cavidade oral para a faringe $\mathrm{e}^{(48,49)}$.

O escape oral anterior de alimento ou líquido após a captação do bolo ${ }^{(42)}$ pode ser causado pela insuficiência do vedamento labial, disfunção que, quando acompanhada da lingual, é variável de acordo com as características individuais ${ }^{(47)}$ e pode, também, favorecer o escape extraoral de saliva, além de, ao longo do tempo, dificultar a contração e elevação da laringe para a deglutição espontânea de saliva ${ }^{(42,50)}$.

Embora nesta revisão de literatura apenas um estudo tenha apresentado resultados relacionados à xerostomia, a sensação de boca seca, quando presente, pode ter como causa o aumento do número de medicamentos administrados, principalmente antipsicóticos, antidepressivos, antiparkinsonianos e anticolinérgicos, necessários para as condições de saúde que surgem ao longo do envelhecimento. Esse sintoma, quando acompanhado da hipofunção cognitiva e sintomas extrapiramidais, pode contribuir para o estabelecimento de quadros de disfagia ${ }^{(48,51-53)}$.

A presença de dificuldade na mastigação pode ser decorrente das alterações da estrutura e função em idosos, como redução na força mastigatória e fadiga muscular causadas por hipotonia dos músculos da mastigação, que propiciam um preparo mais lento do bolo alimentar e podem ocasionar desconfortos durante o processo mastigatório ${ }^{(53)}$, além de dificultar a ingestão dos alimentos sólidos, o que requer a ingestão de líquidos para facilitar a passagem do bolo alimentar ${ }^{(47)}$. Dentre outras condições que complicam a eficiência mastigatória, releva-se a presença de alterações dentárias e/ou próteses mal adaptadas ${ }^{(53)}$, pois o suporte dentário é necessário para manter a motricidade orofacial adequada ${ }^{(54)}$. Todavia, o idoso, mesmo com queixas relacionadas a dificuldades de mastigação, pode realizar adaptações individuais e manter o desempenho no processo mastigatório e posterior deglutição ${ }^{(55)}$.

Diante dos sinais e sintomas discutidos, notou-se que a percepção desses desfechos se torna difícil para o idoso, por acreditar que fazem parte do envelhecimento. Por outro lado, os profissionais de saúde e cuidadores, além do fonoaudiólogo, devem estar atentos para qualquer sinal que alerte para um provável transtorno de deglutição que possa comprometer o estado geral de saúde, não só pelas consequências que traz para a manutenção do estado nutricional, de hidratação e de saúde pulmonar, mas porque acarretam risco de morte e perda na qualidade de vida. Para isso, a calibração da equipe nas ILPIs torna-se fundamental e imprescindível e é uma realidade em outras áreas ${ }^{(56)}$ e cenários ${ }^{(26)}$.

Na interpretabilidade dos riscos de viés, os estudos demonstraram deficiências em alguns aspectos, que evidenciaram presença de fatores de confusão em relação à idade, distribuição por sexo, percepção da doença e condição de saúde e que podem distorcer os resultados quanto à frequência dos sinais e sintomas de DO. Essa falha metodológica poderia ter sido sanada se os estudos apresentassem critérios de análise mais claros e coesos para responderem suas perguntas de pesquisa.

Dentre as limitações do estudo, está o destaque dos artigos apenas para os desfechos de prevalência de DO e seus fatores de risco associados, sem maiores análises sobre o detalhamento dos sinais e sintomas que poderiam resultar em um transtorno da deglutição em idosos que residem em ILPIs. Observaram-se, também, pesquisas com casuística pequena que dificultaram a melhor caracterização dos sinais e sintomas de DO, com metodologias que não apresentaram grupos de comparação para o controle dos resultados e dos fatores de confusão em relação à presença de doenças de base ou sem presença de doenças, nos idosos que se mantiveram com bom estado de saúde. Portanto, como não houve uma diferenciação dos sinais e sintomas de um distúrbio de deglutição, associados, ou não, às patologias de base $\mathrm{e}$, em decorrência da casuística pequena 
e das falhas metodológicas, a interpretação dos achados deve ser analisada com cautela, pois os desfechos encontrados não podem ser generalizados.

\section{CONCLUSÃO}

De acordo com os estudos revisados, os sinais e sintomas mais frequentes relacionados à DO nos idosos institucionalizados foram presença de tosse e engasgo, antes, durante ou após a deglutição, seguidos por pressão de língua diminuída, voz molhada, perda de peso, deglutição lenta, babar, dificuldade na mastigação e demora em completar as refeições. A maioria das pesquisas se enquadrou entre médio e alto risco de viés.

\section{REFERÊNCIAS}

1. Abreu TA, Fernandes-Eloi J, Sousa AMBD. Reflexões acerca dos Impactos Psicossociais da Institucionalização de Idosos no Brasil. Rev Kairós Gerontol. 2017;20(2):333-52. http://dx.doi.org/10.23925/2176901X.2017v20i2p333-352.

2. Camarano AA, Kanso S. As instituições de longa permanência para idosos no Brasil. Rev Bras Estud Popul. 2010;27(1):233-5. http:// dx.doi.org/10.1590/S0102-30982010000100014.

3. Bessa MEP, Silva MJ. Motivações para o ingresso dos idosos em instituições de longa permanência e processos adaptativos: um estudo de caso. Texto Context - Enferm. 2008;17(2):258-65. http://dx.doi. org/10.1590/S0104-07072008000200006.

4. Roeder MA. Segurança Sanitária para instituições de longa permanência para idosos. Lisboa, Portugal: Associação Amigos da Grande Idade; 2009. p. 5-188.

5. Santos BP, Andrade MJC, Silva RO, Menezes EC. Dysphagia in the elderly in long-stay institutions - a systematic literature review. Rev CEFAC. 2018;20(1):123-30. http://dx.doi.org/10.1590/1982021620182013817.

6. Gutierrez SM, Zanato LE, Pelegrini P, Cordeiro RC. Queixas fonoaudiológicas de idosos residentes em uma instituição de longa permanência. Distúrb Comun. 2009;21(1):21-30.

7. Cardoso SV, Olchik MR, Teixeira AR. Alimentação de idosos institucionalizados: relação entre queixas e características sociodemográficas. Distúrb Comun. 2016;28(2):278-85.

8. Magalhães HV Jr, Pernambuco LA, Lima KC, Ferreira MAF. Screening for oropharyngeal dysphagia in older adults: a systematic review of self-reported questionnaires. Gerodontology. 2018;35(3):162-9. http:// dx.doi.org/10.1111/ger.12333.

9. Marik PE, Kaplan D. Aspiration pneumonia and dysphagia in the elderly. Chest. 2003 Jul;124(1):328-36. http://dx.doi.org/10.1378/ chest.124.1.328. PMid:12853541.

10. Brady A. Managing the patient whit dysphagia. Home Healthc Nurse. 2008;26(1):41-6, quiz 47-8. http://dx.doi.org/10.1097/01. NHH.0000305554.40220.6d. PMid:18158492.

11. Roy N, Stemple J, Merrill RM, Thomas L. Dysphagia in the elderly: preliminary evidence of prevalence, risk factors, and socioemotional effects. Ann Otol Rhinol Laryngol. 2007;116(11):858-65. http://dx.doi. org/10.1177/000348940711601112. PMid:18074673.
12. Cardoso SV, Teixeira AR, Baltezan RL, Olchik MR, De Vida Q. O impacto das alterações de deglutição na qualidade de vida de idosos institucionalizados. Rev Kairós Gerontol. 2014;17(1):231-45.

13. Ganong LH. Integrative reviews of nursing research. Res Nurs Health. 1987;10(1):1-11. http://dx.doi.org/10.1002/nur.4770100103. PMid:3644366.

14. Mendeley. Mendeley Ltd. reference manager [Internet]. Amsterdã: Elsevier; 2020 [cited 2021 Dec 20]. Available from: https://www. elsevier.com/pt-br/solutions/mendeley

15. Ouzzani M, Hammady H, Fedorowicz Z, Elmagarmid A. Rayyan-a web and mobile app for systematic reviews. Syst Rev. 2016;5(1):210. http://dx.doi.org/10.1186/s13643-016-0384-4. PMid:27919275.

16. Melnyk BM, Fineout-Overholt E. Evidence-based practice in nursing $\&$ healthcare : a guide to best practice. 2 nd ed. Philadelphia: Wolters Kluwer Health/Lippincott Williams \& Wilkins; 2011. 599 p.

17. Moola S, Munn Z, Tufanaru C, Aromataris E, Sears K, Sfetcu R, et al. Chapter 7: Systematic reviews of etiology and risk. In: Aromataris E, Munn Z, editors. JBI Manual for Evidence Synthesis. JBI; 2020. https://doi.org/10.46658/JBIMES-20-08.

18. Roque FP, Bomfim FMS, Chiari BM. Descrição da dinâmica de alimentação de idosas institucionalizadas. Rev Soc Bras Fonoaudiol. 2010;15(2):256-63. http://dx.doi.org/10.1590/S1516-80342010000200018.

19. Brochier CW, Hugo FN, Rech RS, Baumgarten A, Hilgert JB. Influence of dental factors on oropharyngeal dysphagia among recipients of long-term care. Gerodontology. 2018 Dez;35(4):333-8. http://dx.doi. org/10.1111/ger.12345. PMid:29882353.

20. Siebens H, Trupe E, Siebens A, Cook F, Anshen S, Hanauer R, et al. Correlates and consequences of eating dependency in institutionalized elderly. J Am Geriatr Soc. 1986 Mar;34(3):192-8. http://dx.doi. org/10.1111/j.1532-5415.1986.tb04202.x. PMid:3950287.

21. Nomura Y, Tsutsumi I, Nagasaki M, Tsuda H, Koga F, Kashima N, et al. Supplied food consistency and oral functions of institutionalized elderly. Int J Dent. 2020;3463056. http://dx.doi.org/10.1155/2020/3463056.

22. Gilmore-Bykovskyi AL, Rogus-Pulia N. Temporal associations between caregiving approach, behavioral symptoms and observable indicators of aspiration in nursing home residents with dementia. J Nutr Health Aging. 2018;22(3):400-6. http://dx.doi.org/10.1007/ s12603-017-0943-y. PMid:29484354.

23. Huppertz VAL, Halfens RJG, van Helvoort A, de Groot LCPGM, Baijens LWJ, Schols JMGA. Association between oropharyngeal dysphagia and malnutrition in dutch nursing home residents: results of the national prevalence measurement of quality of care. J Nutr Health Aging. 2018;22(10):1246-52. http://dx.doi.org/10.1007/s12603-0181103-8. PMid:30498833.

24. Ekberg O, Hamdy S, Woisard V, Wuttge-Hannig A, Ortega P. Social and psychological burden of dysphagia: its impact on diagnosis and treatment. Dysphagia. 2002;17(2):139-46. http://dx.doi.org/10.1007/ s00455-001-0113-5. PMid:11956839.

25. Kayser-Jones J, Pengilly K. Dysphagia among nursing home residents. Geriatr Nurs. 1999;20(2):77-82, quiz 84. http://dx.doi.org/10.1053/ gn.1999.v20.97011. PMid:10382421.

26. Lin L-C, Wu S-C, Chen HS, Wang T-G, Chen M-Y. Prevalence of impaired swallowing in institutionalized older people in taiwan. J Am Geriatr Soc. 2002 Jun;50(6):1118-23. http://dx.doi.org/10.1046/j.15325415.2002.50270.x. PMid:12110075.

27. Namasivayam-MacDonald AM, Morrison JM, Steele CM, Keller H. How swallow pressures and dysphagia affect malnutrition and mealtime outcomes in long-term care. Dysphagia. 2017;32(6):785-96. http:// dx.doi.org/10.1007/s00455-017-9825-z. PMid:28733775. 
28. Nogueira D, Reis E. Swallowing disorders in nursing home residents: how can the problem be explained? Clin Interv Aging. 2013;8:221-7. http://dx.doi.org/10.2147/CIA.S39452. PMid:23449951.

29. Park Y-H, Han H-R, Oh B-M, Lee J, Park J, Yu SJ, et al. Prevalence and associated factors of dysphagia in nursing home residents. Geriatr Nurs. 2013;34(3):212-7. http://dx.doi.org/10.1016/j.gerinurse.2013.02.014. PMid:23528180.

30. Steele CM, Greenwood C, Ens I, Robertson C, Seidman-Carlson R. Mealtime difficulties in a home for the aged: not just dysphagia. Dysphagia. 1997;12(1):43-50, discussion 51. http://dx.doi.org/10.1007/ PL00009517. PMid:8997832.

31. Yoshida M, Kikutani T, Tsuga K, Utanohara Y, Hayashi R, Akagawa Y. Decreased tongue pressure reflects symptom of dysphagia. Dysphagia. 2006 Jan;21(1):61-5. http://dx.doi.org/10.1007/s00455-005-9011-6. PMid:16544085.

32. Namasivayam AM, Steele CM, Keller $\mathrm{H}$. The effect of tongue strength on meal consumption in long term care. Clin Nutr. 2016;35(5):107883. http://dx.doi.org/10.1016/j.clnu.2015.08.001. PMid:26321499.

33. Jukic Peladic N, Orlandoni P, Dell'Aquila G, Carrieri B, Eusebi P, Landi $F$, et al. Dysphagia in nursing home residents: management and outcomes. J Am Med Dir Assoc. 2019 Fev;20(2):147-151. PMid:30249360.

34. Davim RM, Torres GV, Dantas SM, Lima VM. Estudo com idosos de instituições asilares no município de Natal/RN: características socioeconômicas e de saúde. Rev Lat Am Enfermagem. 2004;12(3):518-24. http://dx.doi.org/10.1590/S0104-11692004000300010. PMid:15303209.

35. Alcântara RKL, Cavalcante MLSN, Fernandes BKC, Lopes VM, Leite SFP, Borges CL. Perfil sociodemográfico e de saúde de idosos institucionalizados. Rev Enferm UFPE on line. 2019;13(3):674-9. http://dx.doi.org/10.5205/1981-8963-v13i3a237384p674-679-2019.

36. Rech RS, Baumgarten A, Colvara BC, Brochier CW, de Goulart BNG, Hugo FN, et al. Association between oropharyngeal dysphagia, oral functionality, and oral sensorimotor alteration. Oral Dis. 2018;24(4):66472. http://dx.doi.org/10.1111/odi.12809. PMid:29164750.

37. Pu D, Murry T, Wong MCM, Yiu EML, Chan KMK. Indicators of dysphagia in aged care facilities. J Speech Lang Hear Res. 2017 Set;60(9):2416-26. http://dx.doi.org/10.1044/2017 JSLHR-S-17-0028. PMid:28806819.

38. Cabre M, Serra-Prat M, Palomera E, Almirall J, Pallares R, Clavé P. Prevalence and prognostic implications of dysphagia in elderly patients with pneumonia. Age Ageing. 2010;39(1):39-45. http://dx.doi. org/10.1093/ageing/afp100. PMid:19561160.

39. Leder SB, Espinosa JF. Aspiration risk after acute stroke: comparison of clinical examination and fiberoptic endoscopic evaluation of swallowing. Dysphagia. 2002;17(3):214-8. http://dx.doi.org/10.1007/ s00455-002-0054-7. PMid:12140648.

40. Rozenfeld M. A percepção do engasgo por idosos institucionalizados. Estud Interdiscip sobre o Envelhec. 2005;7:69-85. http://dx.doi. org/10.22456/2316-2171.4758.

41. Rozenfeld M, Friedman S. A percepção subjetiva do engasgo em pessoas idosas. Rev Bras Ciências do Envelhec. 2005;2(2):47-56. https://doi.org/10.5335/rbceh.2012.33.

42. Nishiwaki K, Tsuji T, Liu M, Hase K, Tanaka N, Fujiwara T. Identification of a simple screening tool for dysphagia in patients with stroke using factor analysis of multiple dysphagia variables. J Rehabil Med.
2005;37(4):247-51. http://dx.doi.org/10.1080/16501970510026999. PMid:16024482.

43. Kagaya H, Saitoh E, Yokoyama M, Shibata S, Aoyagi Y, Kanamori D, et al. Initiation of pharyngeal response during discrete swallowing and chewswallowing in healthy subjects. Prog Rehabil Med. 2016;1:20160002. http://dx.doi.org/10.2490/prm.20160002. PMid:32789199.

44. Ney DM, Weiss JM, Kind AJH, Robbins JA. Senescent swallowing: impact, strategies and interventions. Nutr Clin Pract. 2010;24(3):395413. http://dx.doi.org/10.1177/0884533609332005. PMid:19483069.

45. Padovani ARP, Moraes DP, Mangili LD, Andrade CRF. Protocolo Fonoaudiológico de Avaliação do Risco para Disfagia (PARD). Rev Soc Bras Fonoaudiol. 2007;12(3):199-205. http://dx.doi.org/10.1590/ S1516-80342007000300007.

46. Lacourt MX, Marini LL. Decréscimo da função muscular decorrente do envelhecimento e a influência na qualidade de vida do idoso: uma revisão de literatura. Rev Bras Ciências do Envelhec Hum. 2006;114-21.

47. Dias BKP, Cardoso MCAF. Características da função de deglutição em um grupo de idosas institucionalizadas. Estud Interdiscip sobre o Envelhec. 2009;14(1):107-24.

48. Clark HM, Henson PA, Barber WD, Stierwalt JAG, Sherrill M. Relationships among subjective and objective measures of tongue strength and oral phase swallowing impairments. Am J Speech Lang Pathol. 2003;12(1):40-50. http://dx.doi.org/10.1044/1058-0360(2003/051). PMid:12680812.

49. Magalhães HV Jr, Tavares JC, Magalhães AAB, Galvão HC, Ferreira MAF. Characterization of tongue pressure in the elderly. Audiol Commun Res. 2014;19(4):375-9.

50. Martins AM S. Avaliação da disfagia: Proposta de Protocolo de Videoendoscopia da Deglutição (VED) [Trabalho de licenciatura]. Porto: Universidade Fernando Pessoa; 2016.

51. Matsumura E, Nohara K, Tanaka N, Fujii N, Sakai T. A survey on medications received by elderly persons with dysphagia living at home or in a nursing home. J Oral Sci. 2020;62(2):239-41. http://dx.doi. org/10.2334/josnusd.19-0370. PMid:32224575.

52. Bomfim FMS, Chiari BM, Roque FP. Fatores associados a sinais sugestivos de disfagia orofaríngea em idosas institucionalizadas. CoDAS. 2013;25(2):154-63. http://dx.doi.org/10.1590/S231717822013000200011. PMid:24408245.

53. Suzuki HS. O entardecer da deglutição: um estudo sobre modificações nos hábitos da rotina alimentar $\mathrm{x}$ fisiologia da deglutição do idoso normal [monografia]. São Paulo (SP): CEFAC - Pós-Graduação em Saúde e Educação; 1997.

54. Pu D, Murry T, Wong MCM, Yiu EML, Chan KMK. Indicators of dysphagia in aged care facilities. J Speech Lang Hear Res. 2017;60(9):2416-26. http://dx.doi.org/10.1044/2017_JSLHR-S-17-0028. PMid:28806819.

55. de Almeida ST, Gentil BC, Nunes EDL. Alterações Miofuncionais Orofaciais Associadas ao Processo de Envelhecimento em um Grupo de Idosos Institucionalizados. Rev Bras Ciências do Envelhec Hum. 2012;9(2):282-92.

56. Lindroos EK, Saarela RKT, Suominen MH, Muurinen S, Soini H, Kautiainen H, et al. Burden of Oral Symptoms and Its Associations With Nutrition, Well-Being, and Survival Among Nursing Home Residents. J Am Med Dir Assoc. 2019;20(5):537-43. http://dx.doi. org/10.1016/j.jamda.2018.10.025. PMid:30541688. 